\title{
A Study on the Model of Structure Analysis of Influence Factors about the Selection of Trip Modes
}

\author{
Shangyun Chen ${ }^{1}$ Shilian Gao ${ }^{2}$ \\ ${ }^{1}$ Department of Mathematical, Southwest Jiaotong Uuiversity, Chengdu 610031, China \\ ${ }^{2}$ College of Traffic and Transportation, Southwest Jiaotong Uuiversity, Chengdu 610031, China
}

\begin{abstract}
The essence of studies on development strategy for urban transportation is the establishment on the attitude to various types of urban transportation mode, which is based on the selection of the trip mode. The oriented graph among all the main influence factors is constructed under the summary of the various influence factors of the selected trip mode. Then adjacency matrixes and reachable matrixes are obtained, and eventually the hierarchical level structure analysis model for the selection process of the trip mode of residents is established. This may contribute to future establishment of urban transportation development strategies.
\end{abstract}

Keywords: Mode of trip, Influence factors, Reachable matrix, Structure analysis, Model

\section{Introduction}

The establishment of development strategies for urban transportations aims to enact the policies and methods which can harmonize the relationship of various transportation modes on the basis of considering the real conditions of each city, so that various types of urban transportation modes may achieve rational structures and advantages of being complementary and eventully satisfy the trip inclinations of the residents. The essence of it is the attitudes towards the various types of urban transportation modes. Therefore, the selection of the trip modes, namely, the selection of transportation modes, is the foundation and an important part on the study development strategies of big cities. It is also an important problem on the theory study and applications of urban transportations ${ }^{[1-6]}$. In order to analyze the process of selection of transportation modes of residents effectively, it is necessary to study the primary factors affecting the selection of transportation modes, especially to distinguish the relating, primary and secondary relationship among the primary influence factors.
Consequently it may contribute to future establishment of urban transportation strategies.

\section{The primary influence factors affecting the selection of trip modes and their relationships}

There are many factors influencing the selection of trip modes and they are also very complicated. But the factors can be summarized and analyzed according to different study angles and emphases. In the mean time, the contradiction of the supply and demand of transportations is the basic pair of contradiction in the development of urban transportations. It plays an important role in the formation and development of urban transportation systems. Therefore, the primary factors influencing selection of trip modes, generally speaking, include the transportation demand factors, the transportation supply factors and the integrated influence factors.

\subsection{Transportation influence factors}

demand

The generation of urban resident trips (including occurrence and attraction) originates from the demand of trips. Such demand is a deriving demand in order to finish certain city activities or acquire certain social services, and achieve a certain trip. Since the trip must satisfy a certain purpose, the tripper do first concern the requiring travel time for arriving the destination after the generation of the trip demand for a purpose. The tripper usually chooses the mode with the shortest trip time when the conditions of trip modes are similar. At the same time, the trip time is equivalent to the distance under several certain conditions. They can transform between each other. With the same trip time, the trip distance is quite different due to different trip modes, vice versa. Moreover, the distribution of trip distances of city residents lies on the using configuration of the urban land (including the natural conditions). 


\subsection{Transportation influence factors}

supply

The demand of transportations accelerates the development of transportation supplies. Contrarily transportation supplies accelerate the further development of transportations. The supplies of urban transportations mainly materialize the collocation of means of transportations, namely, the collocation of the network and its ability, integrated service level of public transportations, technical characteristics of various transportation types. The diversity of collocations of means of transportations makes the selection of trip modes possible. And the growing rate of private cars shows that there is an inevitable trend in the process of the urbanization and urban transportation mechanization, which greatly influences the selection of transportation modes, and eventually greatly impacts the share rate of current transportation modes.

\subsection{Integrated influence factors}

The development level of the urban economy and society and the urban modernization degree directly determine the social economical characters of residents including the professional distribution, income level, consumption intention and the formation of transportation purpose and so on. And they also determine the technical characteristics of various transportation modes including reachability, comfortablility, and safety, which greatly impact the demand and supply of urban transportations. Through the establishment of different transportation development policies and layout measures, we may encourage or limit certain kinds of transportation modes and finally contribute to accelerating or restricting those transportation modes.

In general, there is an interacting and restricting relationship among the influence factors. Primary influence factors of the selection of trip modes of urban residents and their relationship are shown in Fig.1.

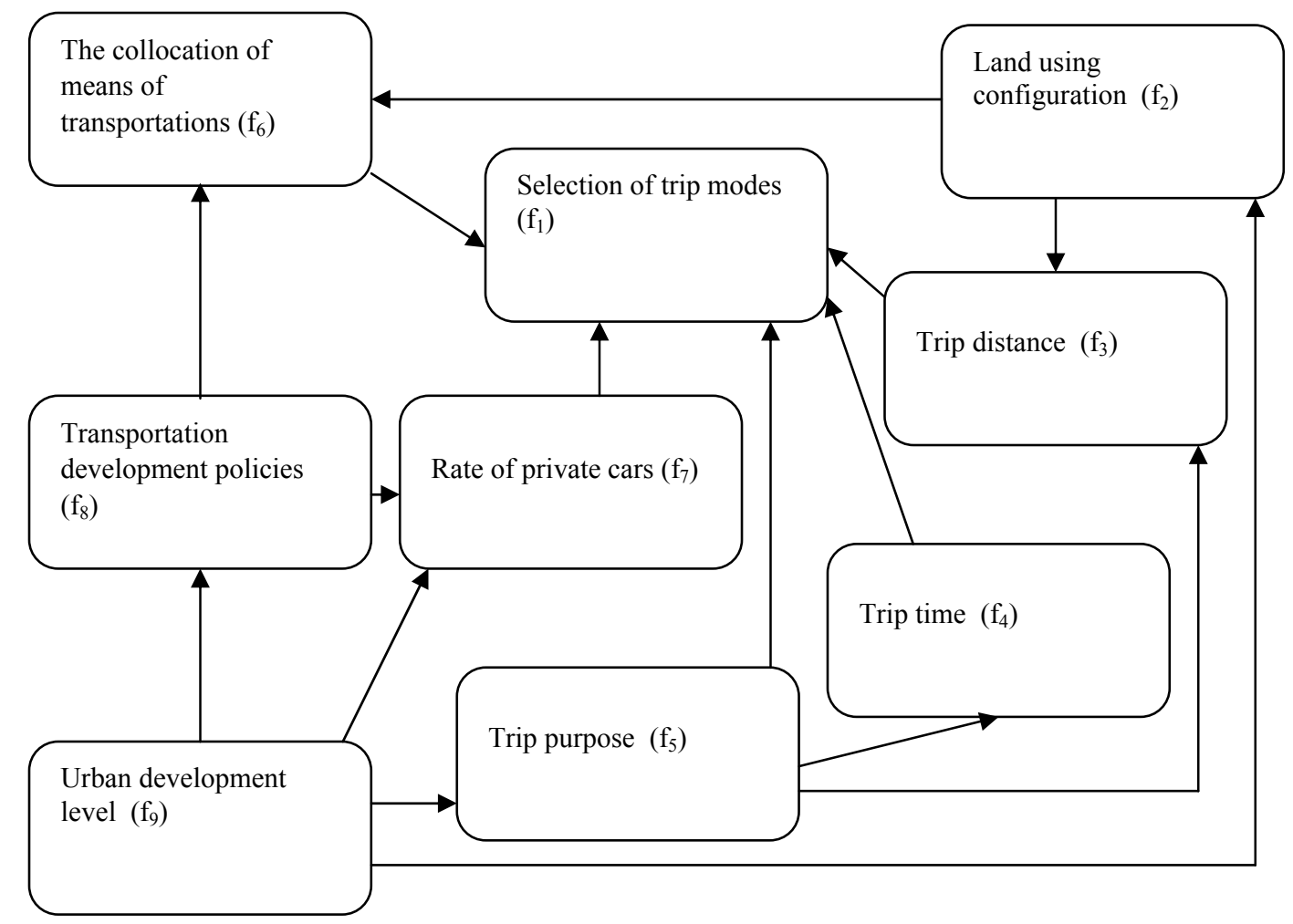

Fig.1 The oriented graph about the influence factors of the selection of trip modes.

\section{The structure analysis model of the main influence factors}

Fig. 1 shows that there exists a complicated relationship among various main influence factors on the selection of trip modes. To obtain a further clear view on it, the structure models that are commonly used in the field of system engineering are applied and 
corresponding operations on the relationship among the various main influence factors are carried on, that is, to establish adjacency matrixes through an oriented graph and then obtain reachable matrixes through adjacency matrixes. Afterwards, we decompose the reachable matrixes and eventually establish a hierarchical level structure analysis model among the main influence factors on the trip $\operatorname{modes}^{[7]}$.

\subsection{Establish adjacency matrixes}

Adjacency matrixes describe the direct relationship between two influence factors. Let $A=\left(\alpha_{i j}\right)$ be an adjacency matrix. Its element $\alpha_{i j}$ is defined as:

$$
\begin{aligned}
a_{i j}=\left\{\begin{array}{l}
1 \text { when } f_{i} \text { can directly reach } f_{j} \\
0 \\
0
\end{array}\right. \\
A=\left[\begin{array}{lllllllllll}
0 & 0 & 0 & 0 & 0 & 0 & 0 & 0 & 0 \\
0 & 0 & 1 & 0 & 0 & 1 & 0 & 0 & 0 \\
1 & 0 & 0 & 0 & 0 & 0 & 0 & 0 & 0 \\
1 & 0 & 0 & 0 & 0 & 0 & 0 & 0 & 0 \\
1 & 0 & 1 & 1 & 0 & 0 & 0 & 0 & 0 \\
1 & 0 & 0 & 0 & 0 & 0 & 0 & 0 & 0 \\
1 & 0 & 0 & 0 & 0 & 0 & 0 & 0 & 0 \\
0 & 0 & 0 & 0 & 0 & 1 & 1 & 0 & 0 \\
0 & 1 & 0 & 0 & 1 & 0 & 1 & 1 & 0
\end{array}\right]
\end{aligned}
$$

\subsection{Calculate reachable matrixes}

Reachable matrixes may be obtained through a series of operations by adjacency matrixes plus identity matrix. It describes the reachablility of various influence factors after a certain length of passages.

$$
\text { Let } A_{1}=(A+I)^{1} \quad, \quad A_{2}=(A+I)^{2}, \quad \ldots \text {, }
$$
$A_{n}=(A+I)^{n}$. Using the operating rule of Boolean algebra, that is, $0+0=0,0+1=1,1+1=1,0 * 0=0,0 * 1=0$, $1 * 1=1$, calculate gradually until $(A+I)^{r}=(A+I)^{r+1}$, we get reachable matrix $M$, namely, $M=A_{2}=A_{3}$.

\subsection{Decomposition of the reachable matrixes}

To analyze the reachable matrixes, first we decompose in the regions, then decompose among classes. The factors related to factor $f_{i}$ constitute the reachability set $X\left(f_{i}\right)$, The factors which may reach the factor $f_{i}$ constitute the antecedent set $Y\left(f_{i}\right)$, that is

$$
X\left(f_{i}\right)=\left\{f_{j} \in F / m_{i j}=1\right\}, \quad Y\left(f_{i}\right)=\left\{f_{j} \in F / m_{j i}=1\right\}
$$

where $\mathrm{F}$ is the set of the nodes composed by all the factors; $m_{i j}$ is the relative value of node $i$ to node $j$ ( the value is 1 or 0 ).

All the factors that satisfy the conditions that the intersection of the reachability set of all the factors $f_{i}$ and the antecedent set equals to the antecedent set will constitute a new set, which is defined as the common set.

$$
\mathrm{T}=\left\{f_{i} \in \mathrm{F} / \mathrm{X}\left(f_{i}\right) \cap \mathrm{Y}\left(f_{i}\right)=\mathrm{Y}\left(f_{i}\right)\right\}
$$

Then based on the known reachable matrix $M$ and formula (1), (2), we obtain the reachability set, the antecedent set and the intersection of the reachability set and the antecedent set shown in Tab. 1.

Then we get $\mathrm{T}=\left\{f_{9}\right\}$. According to the condition of decomposition among classes: $X\left(f_{i}\right) \cap Y\left(f_{i}\right)=X\left(f_{i}\right)$ and from Tab. 1 , only factor $f_{1}$ satisfy this condition, then it is the first class $\mathrm{L}_{1}=\left\{f_{1}\right\}$.

\begin{tabular}{ll|ll}
\hline$i$ & $X\left(f_{i}\right)$ & $Y\left(f_{i}\right)$ & $\begin{array}{c}X\left(f_{i}\right) \\
Y\left(f_{i}\right)\end{array}$ \\
\hline 1 & 1 & $1,2,3,4,5,6,7,8,9$ & 1 \\
\hline 2 & $1,2,3,6$ & 2,9 & 2 \\
\hline 3 & 1,3 & $2,3,5,9$ & 3 \\
\hline 4 & 1,4 & $4,5,9$ & 4 \\
\hline 5 & $1,3,4,5$ & 5,9 & 5 \\
\hline 6 & 1,6 & $2,6,8,9$ & 6 \\
\hline 7 & 1,7 & $7,8,9$ & 7 \\
\hline 8 & $1,6,7,8$ & 8,9 & 8 \\
\hline 9 & $1,2,3,4,5,6,7,8,9$ & 9 & 9 \\
\hline \multicolumn{4}{c}{ Tab.1 Decomposition among class(first } \\
\multicolumn{4}{c}{ class). } \\
\hline \multicolumn{4}{c}{}
\end{tabular}

Remove factor $f_{1}$ from Tab. 1, we get Tab. 2 of decomposition among classes:

\begin{tabular}{ll|ll}
\hline$i$ & $X\left(f_{i}\right)$ & $Y\left(f_{i}\right)$ & $X\left(f_{i} \cap Y\left(f_{i}\right)\right.$ \\
\hline 2 & $2,3,6$ & 2,9 & 2 \\
\hline 3 & 3 & $2,3,5,9$ & 3 \\
\hline 4 & 4 & $4,5,9$ & 4 \\
\hline 5 & $3,4,5$ & 5,9 & 5 \\
\hline 6 & 6 & $2,6,8,9$ & 6 \\
\hline 7 & 7 & $7,8,9$ & 7 \\
\hline 8 & $6,7,8$ & 8,9 & 8 \\
\hline 9 & $2,3,4,5,6,7,8,9$ & 9 & 9 \\
\hline \multicolumn{3}{|c}{ Tab.2 Decomposition among class } \\
\hline
\end{tabular}

Similiarly, we get the second class $L_{2}=\left\{f_{3}, f_{4}\right.$, $\left.f_{6}, f_{7}\right\}$. Also, we can obtain Tab. 3 of decomposition among classes and the third class $L_{3}=\left\{f_{2}, f_{5}, f_{8}\right\}$ and the fourth class $L_{4}=\left\{f_{9}\right\}$. 


\begin{tabular}{ll|ll}
\hline$i$ & $X\left(f_{i}\right)$ & $Y\left(f_{i}\right)$ & $X\left(f_{i} \cap Y\left(f_{i}\right)\right.$ \\
\hline 2 & 2 & 2,9 & 2 \\
\hline 5 & 5 & 5,9 & 5 \\
\hline 8 & 8 & 8,9 & 8 \\
\hline 9 & $2,5,8,9$ & 9 & 9 \\
\hline \multicolumn{4}{c}{ Tab.3 Decomposition among class } \\
(third class)
\end{tabular}

Then we get the reachable matrix with changed bits as follows.

$$
M^{\prime}=\begin{array}{r}
1 \\
3 \\
4 \\
2 \\
2 \\
5 \\
8 \\
8 \\
1
\end{array}\left[\begin{array}{lllllllll}
1 & 0 & 0 & 0 & 0 & 0 & 0 & 0 & 0 \\
1 & 1 & 0 & 0 & 0 & 0 & 0 & 0 & 0 \\
1 & 0 & 1 & 0 & 0 & 0 & 0 & 0 & 0 \\
1 & 0 & 0 & 1 & 0 & 0 & 0 & 0 & 0 \\
1 & 0 & 0 & 0 & 1 & 0 & 0 & 0 & 0 \\
1 & 1 & 0 & 1 & 0 & 1 & 0 & 0 & 0 \\
1 & 0 & 0 & 1 & 1 & 0 & 0 & 1 & 0 \\
1 & 1 & 1 & 1 & 1 & 1 & 1 & 1 & 1
\end{array}\right]
$$

\subsection{Establishment of hierarchical level structure analysis model of the main influence factors}

The reachable matrix $M^{\prime}$ is subtracted by the Identity matrix to establish the hierarchical level among each element. From the definition of reachability, the second class $f_{3}, f_{4}, f_{6}, f_{7}$ is only related to $f_{1}$, that is, $f_{3} \rightarrow f_{1}, f_{4} \rightarrow f_{1}, f_{6} \rightarrow f_{1}, f_{7} \rightarrow f_{1}$. Remove the row and column of $f_{1}$ and decide the relationship between the second class and the third class, we know that there are $f_{2}, f_{5}$ and $f_{8}$ in the third class, that is, $f_{2} \rightarrow f_{3}$, $f_{2} \rightarrow f_{6}, f_{5} \rightarrow f_{3}, f_{5} \rightarrow f_{4}, f_{8} \rightarrow f_{6}, f_{8} \rightarrow f_{7} ; \quad$ Remove the row and columnof $f_{3}, f_{4}, f_{6}, f_{7}$ and we know that there are only $f_{9}$ in the fourth class, that is, $f_{9} \rightarrow f_{2}$, $f_{9} \rightarrow f_{5}, f_{9} \rightarrow f_{8}$. Thus we may establish the hierarchical level structure analysis model of the influence factors on the trip mode selection, which is shown in Fig. 2.

\section{Conclusions}

The above demonstration reveals that the trip distance, trip time, the collocation of means of transportations, and the rate of private cars are the direct factors influencing the selection of trip modes. Land using configuration, trip purpose and transportation development policy could have a hierarchical control effect on the selection of trip modes through the trip distance, trip time, the collocation of means of transportations, and the rate of private cars. Moreover, the urban development level influences the land using configuration, trip purpose and transportation development policy directly, which eventually produces a hierarchical control effect on the selection of trip modes. In the mean time, the study on the selection of trip modes is a very complicated work. A hierarchical level structure analysis model of various main influence factors is established on the basis of the macro-analysis of the main influence factors on the selection of trip modes of urban residents. And the purpose of this paper is to provide a methodology and a technical route on a macro scope for the selection of trip modes. In practice, different cities have different conditions, the influence factors of the trip modes are different, and especially the contents of each influence factor is quite different. All of these are directly related to the concrete study of resident trip modes and the establishment of urban transportation development strategies. And it's also a problem that needs our future research.

\section{Acknowledgement}

This work was supported by the science and technology development project of Railway of Ministry of China under the grant No. 2002X046.

\section{References}

[1] J. Pucher, Transportation Trends, Problems, and Policies: An international Perspective, Transportation Research Part A 33: 493-503, 1999.

[2] R. Kakimoto and S. Mizokami, A Transportation Choice Model with Serial Correlation and State Dependency, Proceedings of ICTTS' 2002 Volume One, the American Society of Civil Engineers, pp. 373-380, 2002.

[3] H. Lloyd, F. Flem, et al, Rail transit -the people's choice, Rail age, 9: 71-80,1997.

[4] L. Roger, M. Mackett and M. Edwards, The Impact of New Urban Public Transportation Systems: Will the Expectation Be Met? Transportation Research Part A, 4: 231-245, 1998.

[5] F. Leurent, Sensitivity and Error Analysis of the Dual Criteria Traffic Assignment Model, Transpn Res.-B, 32:189-204, 1998.

[6] X. Niu, W. Wang and Z. Yin, Resarch on Method Passenger Traffic Mode Split Forecast, Journal of Highway and transporation Research and Development, , 3: 75-77, 2004.

[7] X. Chen, H. Tong and S. Gao, Influencing factors analysis of the network scale of the urban rail transit, China Railway Science, 6: 59-62, 2001. 


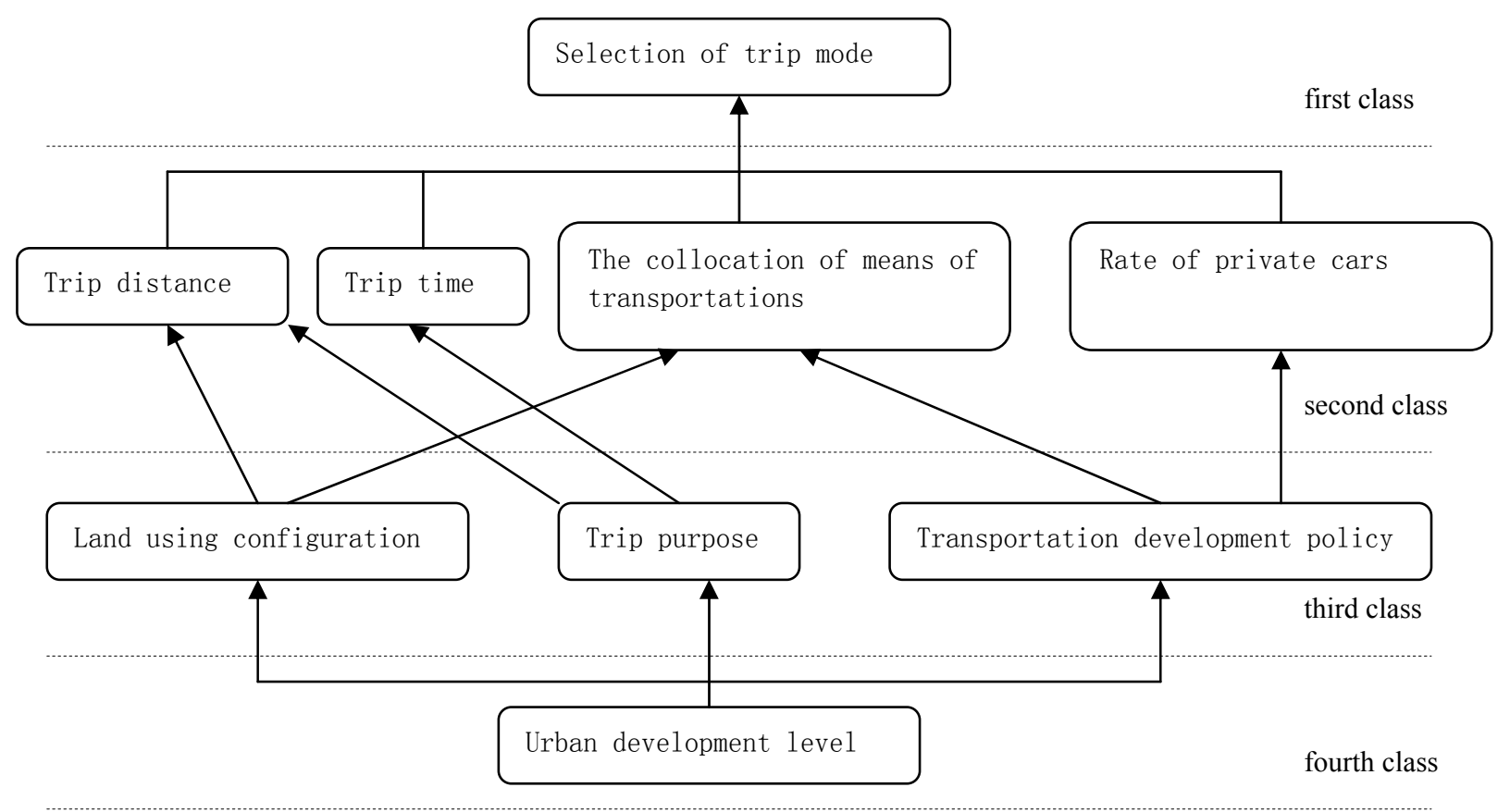

Fig.2 The hierarchical structure analysis model of the influence factors of the selection of trip modes 